Article

\title{
Homogeneous Data Normalization and Deep Learning: A Case Study in Human Activity Classification
}

\author{
Ivan Miguel Pires ${ }^{1,2,3, *(\mathbb{D}}$, Faisal Hussain ${ }^{4}\left(\mathbb{D}\right.$, Nuno M. Garcia ${ }^{1}\left(\mathbb{D}\right.$, Petre Lameski $\left.^{5}{ }^{(}\right)$and \\ Eftim Zdravevski ${ }^{5}$ (D) \\ 1 Instituto de Telecomunicações, Universidade da Beira Interior, 6200-001 Covilhã, Portugal; ngarcia@di.ubi.pt \\ 2 Computer Science Department, Polytechnic Institute of Viseu, 3504-510 Viseu, Portugal \\ 3 UICISA:E Research Centre, School of Health, Polytechnic Institute of Viseu, 3504-510 Viseu, Portugal \\ 4 Department of Computer Engineering, University of Engineering and Technology (UET), Taxila 47080, \\ Pakistan; faisal.hussain.engr@gmail.com \\ 5 Faculty of Computer Science and Engineering, University Ss Cyril and Methodius, 1000 Skopje, \\ North Macedonia; petre.lameski@finki.ukim.mk (P.L.); eftim.zdravevski@finki.ukim.mk (E.Z.) \\ * Correspondence: impires@it.ubi.pt; Tel.: +351-966-379-785
}

Received: 12 October 2020; Accepted: 9 November 2020; Published: 10 November 2020

check for updates

\begin{abstract}
One class of applications for human activity recognition methods is found in mobile devices for monitoring older adults and people with special needs. Recently, many studies were performed to create intelligent methods for the recognition of human activities. However, the different mobile devices in the market acquire the data from sensors at different frequencies. This paper focuses on implementing four data normalization techniques, i.e., MaxAbsScaler, MinMaxScaler, RobustScaler, and Z-Score. Subsequently, we evaluate the impact of the normalization algorithms with deep neural networks (DNN) for the classification of the human activities. The impact of the data normalization was counterintuitive, resulting in a degradation of performance. Namely, when using the accelerometer data, the accuracy dropped from about $79 \%$ to only $53 \%$ for the best normalization approach. Similarly, for the gyroscope data, the accuracy without normalization was about $81.5 \%$, whereas with the best normalization, it was only $60 \%$. It can be concluded that data normalization techniques are not helpful in classification problems with homogeneous data.
\end{abstract}

Keywords: human activities; data normalization; data classification; sensors; mobile devices; data processing

\section{Introduction}

Nowadays, mobile devices in all everyday tasks are increasing, and their usage allows users to stay connected and communicate with ease [1,2]. The current pandemic situation discourages social interaction and personal contacts, enhancing the role of technology in promoting social distancing while being connected [3,4] and active, avoiding sedentary positions [5,6]. Several studies use mobile devices to identify human activities and create a personal agenda to track people [7-10]. This is especially important for people with special needs, including older adults or people with chronic diseases [11-13]. The constant contact with professional healthcare will benefit people's quality of life [14-16].

Sensors are vital for data acquisition related to human activities [17-19] and, lately, even for diagnostic purposes [20,21]. Mobile devices include a large variety of sensors, including accelerometer, magnetometer, gyroscope, acoustic, location, contacts, and other types of sensors [22,23]. 
The development of monitoring solutions is recurrent in the literature, and the advances in this type of device promoted the creation of intelligence solutions [24-30].

The data acquired by sensors depends on the mobile devices' positioning, and their processing is sometimes difficult [31,32]. The data normalization may be powerful in the data processing stage without a large increase in memory and power processing needs [33]. There are different data normalization methods that may be implemented, but the focus of this study was related to the implementation of MaxAbsScaler [34], MinMaxScaler [35], RobustScaler [36], and Z-Score [33] normalizers. Finally, for the data processing, several types of machine learning methods may be implemented, including support vector machine (SVM) [37], decision tree [38], AdaBoost [39,40], artificial neural networks (ANN) [41], k-nearest neighbor (kNN) [42], combined nomenclature (CN2) rule inducer [43], and stochastic gradient descent (SGD) [44]. However, this paper's remainder proposes studying the parameters related to deep neural networks (DNN) [36].

This study aims to explore the use of the "Heterogeneity Activity Recognition Data Set" [2] for the implementation of four data normalization techniques, for further implementation of data classification techniques for the automatic recognition of human activities. Furthermore, what this study aims to achieve is to find out if the used normalization technique influences the human activity recognition performance. The implemented method was based on deep neural networks (DNN) to classify the different data included in the dataset. Furthermore, the data classification results are related to the data normalization methods implemented and compared with the previous studies with other datasets [45-47].

There are many research studies related to the recognition of human activities. Still, it is not possible to say which artificial intelligence method is reliable in general, for any given dataset. This study's scope consists of using data acquired from inertial sensors embedded in a mobile device, including smartphones, smartwatches, and tablets, to implement data normalization and data classification techniques to identify human activities. One lack of previous studies was related to the data acquisition, in that the data were always acquired with the same device, i.e., a BQ Aquaris 5.7 smartphone [48,49].

Previous studies were performed with accelerometer, magnetometer, and gyroscope sensors with the same classification technique implemented in this study $[46,50,51]$. The normalization technique previously implemented was the mean and standard deviation, like the Z-Score normalizer application. The previously used dataset is available at [52].

The previously used dataset has one more human activity than the dataset used in this study. The previous studies used data fusion techniques to merge the accelerometer, magnetometer, and gyroscope sensors' data.

The Introduction section is finished with this paragraph. This paper's remainder is structured as follows: Section 2 proposes the methodology of this study, presenting the dataset, data normalization, peak detection algorithm, feature extraction, data classification, and statistical analysis stages. The different data normalization techniques results are presented in Section 3. This study is concluded in Section 4 with the main conclusions and discussion of the results of this study.

\section{Methods}

Similarly to the previously implemented and published method with artificial intelligence for automatic identification of daily activities [45-47], the data were acquired from off-the-shelf mobile devices. This research implements deep neural networks (DNN), to prove its reliability on the used dataset. The following sections present the details about the dataset used in this research (Section 2.1), and the following sections present the structure proposed at [50]. Section 2.2 continues with the presentation of different data normalization techniques. As in the previous study, this study performed the experimentation of the normalization of the dataset with different normalization techniques. The peak detection algorithm was refined and improved to calculate the features for applying artificial intelligence methods, and it is presented in Section 2.3. Next, the different features extracted are explained in Section 2.4. Next, the data classification methods are applied and presented in Section 2.5. 
Section 2.6 finalizes this study with a comparison of the results. Figure 1 presents the sequence of activities performed for the recognition of human activities.

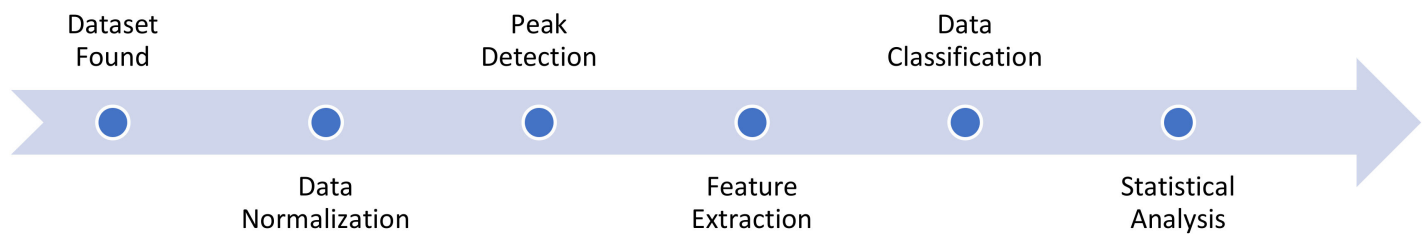

Figure 1. Schema of the data analysis and classification.

\subsection{Dataset}

The dataset used in this research is named the "Heterogeneity Activity Recognition Data Set" [2]. This dataset was acquired from smartphones and smartwatches related to four human activities, including walking upstairs, walking downstairs, standing, and walking. The authors of the dataset reported that it was created to apply machine learning methods for automatic activity recognition. The data acquisition was performed with different mobile devices, including smartwatches, i.e., LG G and Samsung Galaxy Gear, smartphones, i.e., Apple iPhone 6, Samsung Galaxy Pocket+, Samsung Galaxy S3 mini, LG Nexus 4, Samsung Galaxy S3, Samsung Galaxy Nexus, Samsung Galaxy S+, LG Optimus 2X, HTC Desire, and HTC Nexus One, and tablets, i.e., Samsung Galaxy Tab 10.1. The devices used have different frequencies for data acquisition between 25 and $200 \mathrm{~Hz}$. The recordings were performed by 9 different users from the accelerometer and gyroscope sensors at the highest frequency.

\subsection{Data Normalization}

The data of the dataset were normalized to improve the results on activity recognition with machine learning methods. Four data normalization techniques were applied. Firstly, MaxAbsScaler scales and translates each feature individually by the maximum absolute value in the dataset [34]. Secondly, MinMaxScaler scales and translates each feature individually by the given range on the training set [35]. Thirdly, the RobustScaler removes the median and scales the data according to the quantile range [36]. The interquartile range (IQR) [53] is the range between the 1st quartile (25th quantile) and the 3rd quartile (75th quantile). Finally, Z-Score normalization is a normalizing strategy that avoids the outlier issue [33].

\subsection{Peak Detection}

The detection of the sensors' signal variations and maximum values, commonly named peaks, is important for discretizing the different activities because activities with high intensity have more peaks with high values and low intensities have fewer peaks with low values [54].

The detection of peaks may be performed with different methods. This study used a sequential method, smoothing the sensors' signal and saving only the values where the next and previous values are lower. The process must be executed several times until the iteration where the value of peaks is the minimum, but it retains at least five peaks.

\subsection{Feature Extraction}

The definition of the correct and most reliable features for recognizing human activities is important for the obtention of highly accurate results for the method of the automatic recognition of them. Based on the previous knowledge $[46,51,55]$, and the characteristics of the dataset used in this study, the features extracted from the sensors are as follows:

- Accelerometer: mean, standard deviation, variance, and median values of the measured maximum peaks, and mean, standard deviation, variance, median, maximum, and minimum values of the raw signal; 
- Gyroscope: mean, standard deviation, variance, and median values of the measured maximum peaks, and mean, standard deviation, variance, median, maximum, and minimum values of the raw signal.

After the feature extraction, the data classification techniques may be applied to establish the relations between the features and the human activities.

\subsection{Data Classification}

This stage includes applying the artificial intelligence method to identify the human activities available in the dataset. For this purpose, the deep neural networks (DNN) method was applied similarly to $[46,51]$.

For the training stage, we used a Sigmoid activation function, a learning rate of 0.1 , a maximum of $4 \times 10^{6}$ training iterations, 3 hidden layers, the weight function called Xavier, the implementation of backpropagation, and the use of the $\mathrm{L}_{2}$ regularization method [56].

The cross-validation technique was implemented in the testing stage to measure the validity parameters of the implemented method. The results were statistically analyzed, as explained in Section 2.6.

\subsection{Statistical Analysis}

For evaluating the results obtained with the cross-validation technique implemented, the classification performance scores were measured, such as precision, specificity, accuracy, recall, and F1-Score.

Finally, these results are compared with the results obtained with a previously published dataset [51]. Most of the activities included in the dataset used for comparison were also included in the dataset analyzed in this paper, except that captures were performed with other smartphones. Comparing the frequencies of data acquisition, the two datasets may be compared with measuring this dataset's reliability and implementation.

\section{Results}

This research uses an unbalanced dataset to recognize four human activities, including walking upstairs, walking downstairs, standing, and walking. The following section will present the confusion matrixes and other related parameters, such as accuracy, precision, recall, and F1-Score. True positives are cases where the activity was detected accurately. False positives are the cases where the activity was correctly not detected (another activity was present and detected). False positives are cases where the activity was falsely detected, and false negatives are the cases where the activity was detected but other activities should have been detected. Firstly, accuracy is defined as

$$
\text { Accuracy }=\frac{\text { True Positives }+ \text { True Negatives }}{\text { True Positives }+ \text { True Negatives }+ \text { False Positives }+ \text { False Negatives }}
$$

Secondly, precision is defined as

$$
\text { Precision }=\frac{\text { True Positives }}{\text { True Positives }+ \text { False Positives }}
$$

Thirdly, recall is defined as

$$
\text { Recall }=\frac{\text { True Positives }}{\text { True Positives }+ \text { False Negatives }}
$$


Finally, F1-Score is defined as

$$
\text { Fl_Score }=\frac{\text { True Positives }}{\text { True Positives }+0.5(\text { False Positives }+ \text { False Negatives })}
$$

The analysis was performed with data acquired by the accelerometer and gyroscope sensors.

\subsection{Normalized Data with MaxAbsScaler}

Initially, MaxAbsScaler was used to normalize the data acquired from the accelerometer data related to the analyzed human activities, in particular, walking upstairs, walking downstairs, standing, and walking. Table 1 presents the confusion matrix related to the experiments performed with the accelerometer sensor included in the dataset used. It was verified that the most correctly identified activities are walking upstairs, walking downstairs, and walking.

Table 1. Confusion matrix for normalized accelerometer data with MaxAbsScaler.

\begin{tabular}{cccccc}
\hline & & \multicolumn{4}{c}{ Predicted Class } \\
\cline { 3 - 6 } & & $\begin{array}{c}\text { Walking } \\
\text { Upstairs }\end{array}$ & $\begin{array}{c}\text { Walking } \\
\text { Downstairs }\end{array}$ & Standing & Walking \\
\hline \multirow{3}{*}{ Actual Class } & walking upstairs & 75 & 116 & 5 & 5 \\
\cline { 2 - 6 } & walking downstairs & 53 & 141 & 2 & 6 \\
\cline { 2 - 6 } & standing & 8 & 11 & 7 & 12 \\
\cline { 2 - 6 } & walking & 0 & 4 & 3 & 32 \\
\hline
\end{tabular}

Next, the classification results of the data acquired from the accelerometer sensor after the application of MaxAbsScaler were analyzed, verifying that the DNN method implemented reported an accuracy of $53.12 \%$, a precision of $51.59 \%$, a recall value of $51.90 \%$, and an F1-Score of $51.74 \%$.

In continuation, MaxAbsScaler was used to normalize the data acquired from the gyroscope data related to the different human activities. Table 2 presents the confusion matrix related to the experiments performed with the gyroscope sensor included in the dataset used. It was also verified that the most correctly identified activities are walking upstairs, walking downstairs, and walking.

Table 2. Confusion matrix for normalized gyroscope data with MaxAbsScaler.

\begin{tabular}{ccccccc}
\hline & & \multicolumn{4}{c}{ Predicted Class } \\
\cline { 3 - 6 } & & $\begin{array}{c}\text { Walking } \\
\text { Upstairs }\end{array}$ & $\begin{array}{c}\text { Walking } \\
\text { Downstairs }\end{array}$ & Standing & Walking \\
\hline \multirow{3}{*}{ Actual Class } & walking upstairs & 95 & 46 & 0 & 3 \\
\cline { 2 - 6 } & walking downstairs & 52 & 81 & 0 & 11 \\
\cline { 2 - 6 } & standing & 5 & 1 & 7 & 15 \\
\cline { 2 - 6 } & walking & 2 & 4 & 0 & 23 \\
\hline
\end{tabular}

Besides, the classification results of the data acquired from the gyroscope sensor after the application of MaxAbsScaler were analyzed, verifying that the DNN method implemented reported an accuracy of $59.71 \%$, a precision of $66.82 \%$, a recall value of $56.63 \%$, and an F1-Score of $61.31 \%$.

\subsection{Normalized Data with MinMaxScaler}

The second data normalization algorithm that was evaluated on the same accelerometry data was MinMaxScaler. Table 3 presents the confusion matrix related to the experiments performed with the 
accelerometer sensor included in the dataset used. It was verified that the most correctly identified activities are walking downstairs and standing.

Table 3. Confusion matrix for normalized accelerometer data with MinMaxScaler.

\begin{tabular}{lcccccc}
\hline & & \multicolumn{4}{c}{ Predicted Class } \\
\cline { 3 - 6 } & & Walking & $\begin{array}{c}\text { Walking } \\
\text { Downstairs }\end{array}$ & Standing & Walking \\
\hline \multirow{3}{*}{ Actual Class } & walking upstairs & 42 & 153 & 2 & 4 \\
\cline { 2 - 6 } & walking downstairs & 50 & 139 & 12 & 1 \\
\cline { 2 - 6 } & standing & 2 & 8 & 22 & 6 \\
\cline { 2 - 6 } & walking & 2 & 4 & 14 & 19 \\
\hline
\end{tabular}

Next, the classification results of the data acquired from the accelerometer sensor after the application of MinMaxScaler were analyzed, verifying that the DNN method implemented reported an accuracy of $46.25 \%$, a precision of $49.20 \%$, a recall value of $49.08 \%$, and an F1-Score of $49.14 \%$.

MinMaxScaler was also used to normalize the data acquired from the gyroscope data related to the different human activities. Table 4 presents the confusion matrix related to the experiments performed with the gyroscope sensor included in the dataset used. It was also verified that the most correctly identified activities are walking upstairs and walking.

Table 4. Confusion matrix for normalized gyroscope data with MinMaxScaler.

\begin{tabular}{ccccccc}
\hline & & \multicolumn{4}{c}{ Predicted Class } \\
\cline { 3 - 6 } & & $\begin{array}{c}\text { Walking } \\
\text { Upstairs }\end{array}$ & $\begin{array}{c}\text { Walking } \\
\text { Downstairs }\end{array}$ & Standing & Walking \\
\hline \multirow{3}{*}{ Actual Class } & walking upstairs & 107 & 33 & 4 & 0 \\
\cline { 2 - 6 } & walking downstairs & 89 & 47 & 8 & 0 \\
\cline { 2 - 6 } & standing & 7 & 3 & 6 & 12 \\
\cline { 2 - 6 } & walking & 1 & 2 & 4 & 22 \\
\hline
\end{tabular}

Further, the classification results of the data acquired from the gyroscope sensor after the application of MinMaxScaler were analyzed, verifying that the DNN method implemented reported an accuracy of $52.75 \%$, a precision of $49.93 \%$, a recall value of $51.06 \%$, and an F1-Score of $50.49 \%$.

\subsection{Normalized Data with RobustScaler}

Thirdly, RobustScaler was also used to normalize the data acquired from the accelerometer. Table 5 presents the confusion matrix related to the experiments performed with the accelerometer sensor included in the dataset used. It was verified that the most correctly identified activities are walking upstairs and standing. 
Table 5. Confusion matrix for normalized accelerometer data with RobustScaler.

\begin{tabular}{ccccccc}
\hline & & \multicolumn{4}{c}{ Predicted Class } \\
\cline { 3 - 6 } & & $\begin{array}{c}\text { Walking } \\
\text { Upstairs }\end{array}$ & $\begin{array}{c}\text { Walking } \\
\text { Downstairs }\end{array}$ & Standing & Walking \\
\hline \multirow{3}{*}{ Actual Class } & walking upstairs & 115 & 76 & 9 & 1 \\
\cline { 2 - 6 } & walking downstairs & 104 & 77 & 20 & 1 \\
\cline { 2 - 6 } & standing & 4 & 0 & 28 & 6 \\
\cline { 2 - 6 } & walking & 0 & 0 & 20 & 19 \\
\hline
\end{tabular}

Next, the classification results of the data acquired from the accelerometer sensor after the application of RobustScaler were analyzed, verifying that the DNN method implemented reported an accuracy of $49.79 \%$, a precision of $52.16 \%$, a recall value of $54.43 \%$, and an F1-Score of $53.27 \%$.

RobustScaler was used to normalize the data acquired from the gyroscope data related to the different human activities. Table 6 presents the confusion matrix related to the experiments performed with the gyroscope sensor included in the dataset used. It was also verified that the most correctly identified activity is walking upstairs.

Table 6. Confusion matrix for normalized gyroscope data with RobustScaler.

\begin{tabular}{lcccccc}
\hline & & \multicolumn{4}{c}{ Predicted Class } \\
\cline { 3 - 6 } & & Walking & $\begin{array}{c}\text { Walking } \\
\text { Downstairs }\end{array}$ & Standing & \multirow{2}{*}{ Walking } \\
\hline \multirow{3}{*}{ Actual Class } & walking upstairs & 123 & 12 & 1 & 8 \\
\cline { 2 - 6 } & walking downstairs & 97 & 40 & 0 & 7 \\
\cline { 2 - 6 } & standing & 15 & 1 & 5 & 7 \\
\cline { 2 - 6 } & walking & 15 & 1 & 5 & 7 \\
\hline
\end{tabular}

Further, the classification results of the data acquired from the gyroscope sensor after the application of RobustScaler were analyzed, verifying that the DNN method implemented reported an accuracy of $50.87 \%$, a precision of $48.22 \%$, a recall value of $39.01 \%$, and an F1-Score of $43.13 \%$.

\subsection{Normalized Data with Z-Score}

The last data normalization approach that was evaluated was the Z-Score normalizer. Table 7 presents the confusion matrix related to the experiments performed with the accelerometer sensor included in the dataset used. It was verified that the most correctly identified activities are walking upstairs and walking.

Next, the classification results of the data acquired from the accelerometer sensor after the application of the Z-Score normalizer were analyzed, verifying that the DNN method implemented reported an accuracy of $52.71 \%$, a precision of $59.04 \%$, a recall value of $48.07 \%$, and an F1-Score of $52.99 \%$. 
Table 7. Confusion matrix for normalized accelerometer data with Z-Score.

\begin{tabular}{ccccccc}
\hline & & \multicolumn{4}{c}{ Predicted Class } \\
\cline { 3 - 6 } & & $\begin{array}{c}\text { Walking } \\
\text { Upstairs }\end{array}$ & $\begin{array}{c}\text { Walking } \\
\text { Downstairs }\end{array}$ & Standing & Walking \\
\hline \multirow{3}{*}{ Actual Class } & walking upstairs & 167 & 33 & 1 & 0 \\
\cline { 2 - 6 } & walking downstairs & 141 & 54 & 2 & 5 \\
\cline { 2 - 6 } & standing & 12 & 13 & 6 & 7 \\
\cline { 2 - 6 } & walking & 4 & 9 & 0 & 26 \\
\hline
\end{tabular}

In continuation, the Z-Score normalizer was used to normalize the data acquired from the gyroscope data related to the different human activities. Table 8 presents the confusion matrix related to the experiments performed with the gyroscope sensor included in the dataset used. It was also verified that the most correctly identified activities are walking upstairs, standing, and walking.

Table 8. Confusion matrix for normalized gyroscope data with Z-Score.

\begin{tabular}{ccccccc}
\hline & & \multicolumn{4}{c}{ Predicted Class } \\
\cline { 3 - 6 } & & Walking & $\begin{array}{c}\text { Walking } \\
\text { Downstairs }\end{array}$ & Standing & Walking \\
\hline \multirow{3}{*}{ Actual Class } & walking upstairs & 117 & 16 & 1 & 0 \\
\cline { 2 - 6 } & walking downstairs & 89 & 52 & 0 & 3 \\
\cline { 2 - 6 } & standing & 3 & 10 & 15 & 0 \\
\cline { 2 - 6 } & walking & 1 & 9 & 7 & 12 \\
\hline
\end{tabular}

Besides, the classification results of the data acquired from the gyroscope sensor after the application of the Z-Score normalizer were analyzed, verifying that the DNN method implemented reported an accuracy of $56.81 \%$, a precision of $63.63 \%$, a recall value of $53.08 \%$, and an F1-Score of $57.88 \%$.

\subsection{Non-Normalized Data}

Finally, we evaluated the non-normalized data acquired from the accelerometer data related to the four human activities. Table 9 presents the confusion matrix related to the experiments performed with the accelerometer sensor included in the dataset used. It was verified that the most correctly identified activities are walking upstairs, walking downstairs, and walking.

Next, the classification results of the non-normalized data acquired from the accelerometer sensor were analyzed, verifying that the DNN method implemented reported an accuracy of $79.11 \%$, a precision of $78.52 \%$, a recall value of $67.62 \%$, and an F1-Score of $72.66 \%$.

In continuation, the non-normalized data acquired from the gyroscope data related to the different human activities were analyzed. Table 10 presents the confusion matrix related to the experiments performed with the gyroscope sensor included in the dataset used. It was also verified that the most correctly identified activities are walking upstairs, walking downstairs, and walking. 
Table 9. Confusion matrix for non-normalized accelerometer data.

\begin{tabular}{ccccccc}
\hline & & \multicolumn{4}{c}{ Predicted Class } \\
\cline { 3 - 6 } & & Walking & $\begin{array}{c}\text { Walking } \\
\text { Downstairs }\end{array}$ & Standing & \multirow{2}{*}{ Walking } \\
\hline \multirow{3}{*}{ Actual Class } & walking upstairs & 293 & 51 & 2 & 1 \\
\cline { 2 - 6 } & walking downstairs & 54 & 293 & 0 & 0 \\
\cline { 2 - 6 } & standing & 9 & 54 & 3 & 0 \\
\cline { 2 - 6 } & walking & 0 & 2 & 0 & 66 \\
\hline
\end{tabular}

Table 10. Confusion matrix for non-normalized gyroscope data.

\begin{tabular}{cccccc}
\hline & & \multicolumn{4}{c}{ Predicted Class } \\
\cline { 3 - 6 } & & $\begin{array}{c}\text { Walking } \\
\text { Upstairs }\end{array}$ & $\begin{array}{c}\text { Walking } \\
\text { Downstairs }\end{array}$ & Standing & \multirow{2}{*}{ Walking } \\
\hline \multirow{3}{*}{ Actual Class } & walking upstairs & 230 & 21 & 2 & 0 \\
\cline { 2 - 6 } & walking downstairs & 45 & 202 & 6 & 0 \\
\cline { 2 - 6 } & standing & 24 & 12 & 12 & 0 \\
\cline { 2 - 6 } & walking & 0 & 2 & 0 & 48 \\
\hline
\end{tabular}

The classification results of the non-normalized data acquired from the gyroscope sensor were analyzed, verifying that the DNN method implemented reported an accuracy of $81.46 \%$, a precision of $80.54 \%$, a recall value of $72.94 \%$, and an F1-Score of $76.55 \%$.

\subsection{Overall Results}

Based on the results obtained with this study, the best results were achieved with the gyroscope data without applying normalization techniques. It was expected that the use of normalized data would report the best results, but it was not verified, as presented in Figure 2.



Figure 2. Results on the data classification with accelerometer and gyroscope sensors.

Analyzing the accelerometer data, the best accuracy was reported with non-normalized data $(79.11 \%)$, and the application of normalization techniques decreased the accuracy. Firstly, the application of Z-Score normalization decreased the results by $26.4 \%$. Secondly, the application of RobustScaler 
decreased the results by $29.32 \%$. Thirdly, the application of MinMaxScaler decreased the results by $32.86 \%$. Finally, the application of MaxAbsScaler decreased the results by $25.99 \%$.

The gyroscope data analysis revealed that the best accuracy reported was also with non-normalized data $(81.46 \%)$, and the application of normalization techniques also decreased the accuracy. Firstly, the application of Z-Score normalization decreased the results by $24.65 \%$. Secondly, the application of RobustScaler decreased the results by 30.59\%. Thirdly, the application of MinMaxScaler decreased the results by $28.71 \%$. Finally, the application of MaxAbsScaler decreased the results by $21.75 \%$.

\section{Discussion and Conclusions}

The "Heterogeneity Activity Recognition Data Set" [2] was acquired with different mobile devices, including smartphones, tablets, and smartwatches. Different devices have different frequencies of data acquisition. We experimented with four normalization techniques: MaxAbsScaler, MinMaxScaler, RobustScaler, and Z-Score. Furthermore, the DNN method was implemented for the classification of the different human activities.

This study analyzed the difference between non-normalized and normalized data, verifying that the dataset used in this study revealed the best results with the non-normalized data. However, the previously used dataset revealed the best results with normalized data.

The results showed that the best accuracy $(81.46 \%)$ was reported with non-normalized gyroscope data to recognize three human activities. Furthermore, $79.11 \%$ accuracy was obtained with the use of accelerometer data to recognize three human activities, also with non-normalized data. On the contrary, the previously used dataset revealed the best accuracy with normalized data with or without data fusion techniques.

Based on the comparison of the previous results presented in Table 11, non-normalized data reported better accuracy with the dataset analyzed in this study than the previously used dataset. However, normalization techniques reported bad accuracy with this dataset compared to the previously used dataset which reported the correct recognition of five human activities. This dataset only reported a maximum of three human activities correctly.

As future work, the impact of different techniques for data classification, data imputation, and data normalization should be explored, as well as their impact when processing multi-modal data collected by various sensors. Furthermore, other data normalization techniques should be evaluated, as well as how the subsequently used machine learning algorithms benefit from the normalization. As this research shows, deep learning algorithms can overcome bias in the data without normalization. Furthermore, when processing homogeneous data collected by mobile devices, with completely identical data collection frequencies and different ranges of data, this research shows that data normalization impairs the classification accuracy. Other studies $[56,57]$ show that more classical algorithms, such as SVMs, decision trees, and tree ensembles, considerably benefit from data normalization. These algorithms need to be further evaluated with the proposed approaches. In the future, the impact of the presented data normalization and imputation methods should also be evaluated on other datasets. In particular, when using other sensors in collecting multi-modal data from various sensors, such as microphones [58], pressure sensors, infrared sensors, proximity sensors, and oximeters, we expect the impact of the proposed data normalization algorithms to be even more emphasized. 
Table 11. Comparison of results obtained with a previous study.

\begin{tabular}{|c|c|c|c|c|}
\hline Dataset & Sensor(s) & Normalizer & Number of Activities Detected & Accuracy \\
\hline [2] & Accelerometer & MaxAbsScaler & 3 & $53.12 \%$ \\
\hline [2] & Gyroscope & MaxAbsScaler & 3 & $59.71 \%$ \\
\hline [2] & Accelerometer & MinMaxScaler & 2 & $46.25 \%$ \\
\hline [2] & Gyroscope & MinMaxScaler & 2 & $52.75 \%$ \\
\hline [2] & Accelerometer & RobustScaler & 2 & $49.79 \%$ \\
\hline [2] & Gyroscope & RobustScaler & 1 & $50.87 \%$ \\
\hline [2] & Accelerometer & Z-Score & 2 & $52.71 \%$ \\
\hline [2] & Gyroscope & Z-Score & 3 & $56.81 \%$ \\
\hline [2] & Accelerometer & None & 3 & $79.11 \%$ \\
\hline [2] & Gyroscope & None & 3 & $81.46 \%$ \\
\hline [50] & Accelerometer & None & 1 & $22.90 \%$ \\
\hline [50] & Accelerometer & $\begin{array}{c}\text { Mean and } \\
\text { Standard Deviation } \\
\text { (similar to Z-Score) }\end{array}$ & 5 & $85.89 \%$ \\
\hline [49] & $\begin{array}{l}\text { Accelerometer and } \\
\text { Magnetometer }\end{array}$ & None & 2 & $40.69 \%$ \\
\hline [49] & $\begin{array}{l}\text { Accelerometer and } \\
\text { Magnetometer }\end{array}$ & $\begin{array}{c}\text { Mean and } \\
\text { Standard Deviation } \\
\text { (similar to Z-Score) }\end{array}$ & 5 & $86.49 \%$ \\
\hline [49] & $\begin{array}{l}\text { Accelerometer, } \\
\text { Magnetometer, and } \\
\text { Gyroscope }\end{array}$ & None & 4 & $74.46 \%$ \\
\hline [49] & $\begin{array}{c}\text { Accelerometer, } \\
\text { Magnetometer, and } \\
\text { Gyroscope }\end{array}$ & $\begin{array}{l}\text { Mean and } \\
\text { Standard Deviation } \\
\text { (similar to Z-Score) }\end{array}$ & 5 & $89.52 \%$ \\
\hline
\end{tabular}

In conclusion, the benefits of the implementation of data normalization techniques depend on the dataset. It is unclear if normalization would improve the data classification because the number of samples used was smaller than the previously used dataset. As the dataset is unbalanced, it may also influence the implementation of artificial intelligence methods for activity recognition.

Author Contributions: Conceptualization, methodology, software, validation, formal analysis, investigation, writing—original draft preparation, writing—review and editing; I.M.P., F.H., N.M.G., P.L. and E.Z. All authors have read and agreed to the published version of the manuscript.

Funding: This work is funded by FCT/MEC through national funds and co-funded by FEDER_PT2020 partnership agreement under the project UIDB/50008/2020 (Este trabalho é financiado pela FCT/MEC através de fundos nacionais e cofinanciado pelo FEDER, no âmbito do Acordo de Parceria PT2020 no âmbito do projeto UIDB/50008/2020). This work is also funded by National Funds through the FCT-Foundation for Science and Technology, I.P., within the scope of the project UIDB/00742/2020. Furthermore, we would like to thank the Politécnico de Viseu for their support.

Acknowledgments: This work is funded by FCT/MEC through national funds and co-funded by FEDER-PT2020 partnership agreement under the project UIDB/50008/2020 (Este trabalho é financiado pela FCT/MEC através de fundos nacionais e cofinanciado pelo FEDER, no âmbito do Acordo de Parceria PT2020 no âmbito do projeto UIDB/50008/2020). This work is also funded by National Funds through the FCT-Foundation for Science and Technology, I.P., within the scope of the project UIDB/00742/2020. Furthermore, we would like to thank the Politécnico de Viseu for their support. This article is based upon work from COST Action IC1303-AAPELE-Architectures, Algorithms and Protocols for Enhanced Living Environments and COST Action CA16226-SHELD-ON-Indoor living space improvement: Smart Habitat for the Elderly, supported by COST (European Cooperation in Science and Technology). More information at www.cost.eu.

Conflicts of Interest: The authors declare no conflict of interest. 


\section{References}

1. Hung, W.-C.; Shen, F.; Wu, Y.-L.; Hor, M.-K.; Tang, C.-Y. Activity Recognition with sensors on mobile devices. In Proceedings of the 2014 International Conference on Machine Learning and Cybernetics, Lanzhou, China, 13-16 July 2014; pp. 449-454.

2. Stisen, A.; Blunck, H.; Bhattacharya, S.; Prentow, T.S.; Kjærgaard, M.B.; Dey, A.; Sonne, T.; Jensen, M.M. Smart Devices are Different: Assessing and Mitigating: Mobile Sensing Heterogeneities for Activity Recognition. In Proceedings of the 13th ACM Conference on Embedded Networked Sensor Systems-SenSys '15, Seoul, Korea, 1-4 November 2015; ACM Press: Seoul, Korea, 2015; pp. 127-140.

3. Adans-Dester, C.; Bamberg, S.; Bertacchi, F.; Caulfield, B.; Chappie, K.; Demarchi, D.; Erb, M.K.; Estrada, J.; Fabara, E.; Freni, M.; et al. Can mHealth Technology Help Mitigate the Effects of the COVID-19 Pandemic? IEEE Open J. Eng. Med. Biol. 2020, 1. [CrossRef]

4. Zeinalipour-Yazti, D.; Claramunt, C. COVID-19 Mobile Contact Tracing Apps (MCTA): A Digital Vaccine or a Privacy Demolition? In Proceedings of the 2020 21st IEEE International Conference on Mobile Data Management (MDM), Versailles, France, 30 June-3 July 2020; pp. 1-4.

5. Shany, T.; Redmond, S.J.; Narayanan, M.R.; Lovell, N.H. Sensors-Based Wearable Systems for Monitoring of Human Movement and Falls. IEEE Sens. J. 2012, 12, 658-670. [CrossRef]

6. Wong, W.Y.; Wong, M.S.; Lo, K.H. Clinical applications of sensors for human posture and movement analysis: A review. Prosthet. Orthot. Int. 2007, 31, 62-75. [CrossRef] [PubMed]

7. Chen, Y.; Shen, C. Performance Analysis of Smartphone-Sensor Behavior for Human Activity Recognition. IEEE Access 2017, 5, 3095-3110. [CrossRef]

8. Ignatov, A. Real-time human activity recognition from accelerometer data using Convolutional Neural Networks. Appl. Soft Comput. 2018, 62, 915-922. [CrossRef]

9. Tian, Y.; Chen, W. MEMS-based human activity recognition using smartphone. In Proceedings of the 2016 35th Chinese Control Conference (CCC), Chengdu, China, 27-29 July 2016; pp. 3984-3989.

10. Zhu, Q.; Chen, Z.; Soh, Y.C. Smartphone-based Human Activity Recognition in buildings using Locality-constrained Linear Coding. In Proceedings of the 2015 IEEE 10th Conference on Industrial Electronics and Applications (ICIEA), Auckland, New Zealand, 15-17 June 2015; pp. 214-219.

11. Doyle, J.; Kealy, A.; Loane, J.; Walsh, L.; O’Mullane, B.; Flynn, C.; Macfarlane, A.; Bortz, B.; Knapp, R.B.; Bond, R. An integrated home-based self-management system to support the wellbeing of older adults. J. Ambient. Intell. Smart Environ. 2014, 6, 359-383. [CrossRef]

12. Zainal, A.; Razak, F.H.A.; Ahmad, N.A. Older People and the Use of Mobile Phones: An Interview Study. In Proceedings of the 2013 International Conference on Advanced Computer Science Applications and Technologies, Kuching, Malaysia, 23-24 December 2013; pp. 390-395.

13. Tan, Q.L.L.; Chye, L.M.Y.; Ng, D.H.M.; Chong, M.S.; Ng, T.P.; Wee, S.L. Feasibility of a community-based Functional Power Training program for older adults. Clin. Interv. Aging 2018, 13, 309-316. [CrossRef]

14. Guimarães, H.; Boix, H.; Rodrigues, C.; Tomé, T.; Rocha, G.; Vento, M. Impact of the global financial crisis on newborn care in Portugal and Spain: Perception of health professionals. Acta Paediatr. 2020, 109, 625-627. [CrossRef]

15. Por, J.; Barriball, L.; Fitzpatrick, J.; Roberts, J. Emotional intelligence: Its relationship to stress, coping, well-being and professional performance in nursing students. Nurse Educ. Today 2011, 31, 855-860. [CrossRef]

16. Steinhubl, S.R.; Muse, E.D.; Topol, E.J. The emerging field of mobile health. Sci. Transl. Med. 2015, 7, 283 rv3. [CrossRef]

17. Sendra, S.; Granell, E.; Lloret, J.; Rodrigues, J.J.P.C. Smart collaborative system using the sensors of mobile devices for monitoring disabled and elderly people. In Proceedings of the 2012 IEEE International Conference on Communications (ICC), Ottawa, ON, Canada, 10-15 June 2012; pp. 6479-6483.

18. Majumder, S.; Deen, M.J. Smartphone Sensors for Health Monitoring and Diagnosis. Sensors 2019, $19,2164$. [CrossRef] [PubMed]

19. Galán-Mercant, A.; Cuesta-Vargas, A.I. Clinical frailty syndrome assessment using inertial sensors embedded in smartphones. Physiol. Meas. 2015, 36, 1929-1942. [CrossRef] [PubMed]

20. Glowinski, S.; Łosiński, K.; Kowiański, P.; Waśkow, M.; Bryndal, A.; Grochulska, A. Inertial Sensors as a Tool for Diagnosing Discopathy Lumbosacral Pathologic Gait: A Preliminary Research. Diagnostics 2020, 10, 342. [CrossRef] [PubMed] 
21. Godfrey, A.; Bourke, A.K.; Ólaighin, G.M.; van de Ven, P.; Nelson, J. Activity classification using a single chest mounted tri-axial accelerometer. Med. Eng. Phys. 2011, 33, 1127-1135. [CrossRef] [PubMed]

22. Stankevich, E.; Paramonov, I.; Timofeev, I. Mobile phone sensors in health applications. In Proceedings of the 2012 12th Conference of Open Innovations Association (FRUCT), Oulu, Finland, 5-9 November 2012; pp. 1-6.

23. Sousa, P.; Sabugueiro, D.; Felizardo, V.; Couto, R.; Pires, I.; Garcia, N. mHealth sensors and applications for personal aid. In Mobile Health; Springer: Cham, Switzerland, 2015; pp. 265-281.

24. Amoh, J.; Odame, K.M. Technologies for Developing Ambulatory Cough Monitoring Devices. Crit. Rev. Biomed. Eng. 2014. [CrossRef]

25. Appelboom, G.; Camacho, E.; Abraham, M.E.; Bruce, S.S.; Dumont, E.L.; Zacharia, B.E.; D'Amico, R.; Slomian, J.; Reginster, J.Y.; Bruyère, O.; et al. Smart wearable body sensors for patient self-assessment and monitoring. Arch Public Health 2014, 72, 28. [CrossRef] [PubMed]

26. Kakria, P.; Tripathi, N.K.; Kitipawang, P. A Real-Time Health Monitoring System for Remote Cardiac Patients Using Smartphone and Wearable Sensors. Int. J. Telemed. Appl. 2015, 2015, 1-11. [CrossRef]

27. Guo, J.; Zhou, X.; Sun, Y.; Ping, G.; Zhao, G.; Li, Z. Smartphone-Based Patients' Activity Recognition by Using a Self-Learning Scheme for Medical Monitoring. J. Med. Syst. 2016, 40, 140. [CrossRef]

28. Pantelopoulos, A.; Bourbakis, N.G. A survey on wearable sensor-based systems for health monitoring and prognosis. IEEE Trans. Syst. Man Cybern. Part C 2010, 40, 1-12. [CrossRef]

29. Pires, I.M.; Valente, T.; Pombo, N.; Garcia, N.M. Conceptual Definition of a Platform for the Monitoring of the Subjects with Nephrolithiasis Based on the Energy Expenditure and the Activities of Daily Living Performed. In Highlights of Practical Applications of Agents, Multi-Agent Systems, and Complexity: The PAAMS Collection; Springer: Berlin/Heidelberg, Germany, 2018.

30. Shahriyar, R.; Bari, M.F.; Kundu, G.; Ahamed, S.I.; Akbar, M.M. Intelligent Mobile Health Monitoring System (IMHMS). In Electronic Healthcare; Kostkova, P., Ed.; Lecture Notes of the Institute for Computer Sciences, Social Informatics and Telecommunications Engineering; Springer: Berlin/Heidelberg, Germany, 2010; Volume 27, pp. 5-12, ISBN 978-3-642-11744-2.

31. Pires, I.; Felizardo, V.; Pombo, N.; Garcia, N.M. Limitations of energy expenditure calculation based on a mobile phone accelerometer. In Proceedings of the 2017 International Conference on High Performance Computing \& Simulation (HPCS), Genoa, Italy, 17-21 July 2017; pp. 124-127.

32. Pires, I.M.; Garcia, N.M.; Pombo, N.; Flórez-Revuelta, F. Limitations of the Use of Mobile Devices and Smart Environments for the Monitoring of Ageing People. In Proceedings of the 4th International Conference on Information and Communication Technologies for Ageing Well and e-Health (ICT4AWE 2018); Science and Technology Publications, Lda: Setúbal, Portugal, 2018.

33. Patro, S.G.K.; Sahu, K.K. Normalization: A Preprocessing Stage. Int. Adv. Res. J. Sci. Eng. Technol. 2015, 20-22. [CrossRef]

34. Mistry, J.; Inden, B. An Approach to Sign Language Translation using the Intel RealSense Camera. In Proceedings of the 2018 10th Computer Science and Electronic Engineering (CEEC), Colchester, UK, 19-21 September 2018; pp. 219-224.

35. Narkhede, A.H. Human Activity Recognition Based on Multimodal Body Sensing. Master's Thesis, San Jose State University, San Jose, CA, USA, 2019.

36. Vermeulen, A.F. Unsupervised Learning: Deep Learning. In Industrial Machine Learning; Apress: Berkeley, CA, USA, 2020; pp. 225-241, ISBN 978-1-4842-5315-1.

37. Lameski, P.; Zdravevski, E.; Mingov, R.; Kulakov, A. SVM Parameter Tuning with Grid Search and Its Impact on Reduction of Model Over-fitting. In Rough Sets, Fuzzy Sets, Data Mining, and Granular Computing; Yao, Y., Hu, Q., Yu, H., Grzymala-Busse, J.W., Eds.; Lecture Notes in Computer Science; Springer International Publishing: Cham, Switzerland, 2015; Volume 9437, pp. 464-474, ISBN 978-3-319-25782-2.

38. Kamiński, B.; Jakubczyk, M.; Szufel, P. A framework for sensitivity analysis of decision trees. Cent. Eur. J. Oper. Res. 2018, 26, 135-159. [CrossRef] [PubMed]

39. Freund, Y.; Schapire, R.E. A Decision-Theoretic Generalization of On-Line Learning and an Application to Boosting. J. Comput. Syst. Sci. 1997, 55, 119-139. [CrossRef]

40. Hastie, T.; Rosset, S.; Zhu, J.; Zou, H. Multi-class AdaBoost. Stat. Interface 2009, 2, 349-360. [CrossRef]

41. Hassoun, M.H. Fundamentals of Artificial Neural Networks; MIT Press: Cambridge, MA, USA, 1995; ISBN 0-262-08239-X. 
42. Altman, N.S. An Introduction to Kernel and Nearest-Neighbor Nonparametric Regression. Am. Stat. 1992, 46, 175-185. [CrossRef]

43. Clark, P.; Niblett, T. The CN2 induction algorithm. Mach Learn 1989, 3, 261-283. [CrossRef]

44. Pfaff, F.; Noack, B.; Hanebeck, U.D. Data validation in the presence of stochastic and set-membership uncertainties. In Proceedings of the Information Fusion (FUSION), 2013 16th International Conference on Information Fusion, Istanbul, Turkey, 9-12 July 2013; pp. 2125-2132.

45. Pires, I.M.; Teixeira, M.C.; Pombo, N.; Garcia, N.M.; Flórez-Revuelta, F.; Spinsante, S.; Goleva, R.; Zdravevski, E. Android Library for Recognition of Activities of Daily Living: Implementation Considerations, Challenges, and Solutions. Open Bioinform. J. 2018, 11, 61-88. [CrossRef]

46. Pires, I.M.; Garcia, N.M.; Pombo, N.; Flórez-Revuelta, F.; Spinsante, S.; Teixeira, M.C. Identification of activities of daily living through data fusion on motion and magnetic sensors embedded on mobile devices. Pervasive Mob. Comput. 2018, 47, 78-93. [CrossRef]

47. Pires, I.M.; Garcia, N.M.; Pombo, N.; Flórez-Revuelta, F. From Data Acquisition to Data Fusion: A Comprehensive Review and a Roadmap for the Identification of Activities of Daily Living Using Mobile Devices. Sensors 2016, 16, 184. [CrossRef]

48. Smartphones BQ Aquaris | BQ Portugal. Available online: https://www.bq.com/pt/smartphones (accessed on 29 August 2020).

49. BQ Aquaris 5.7-Specifications. Available online: https://www.devicespecifications.com/en/model/59bb30eb (accessed on 17 September 2020).

50. Pires, I.M.; Hussain, F.; Garcia, N.M.; Zdravevski, E. Improving Human Activity Monitoring by Imputation of Missing Sensory Data: Experimental Study. Future Internet 2020, 12, 155. [CrossRef]

51. Pires, I.M.; Marques, G.; Garcia, N.M.; Flórez-Revuelta, F.; Canavarro Teixeira, M.; Zdravevski, E.; Spinsante, S.; Coimbra, M. Pattern Recognition Techniques for the Identification of Activities of Daily Living Using a Mobile Device Accelerometer. Electronics 2020, 9, 509. [CrossRef]

52. Pires, I. Raw dataset with accelerometer, gyroscope and magnetometer data for activities with motion. Mendeley 2020. [CrossRef]

53. Wan, X.; Wang, W.; Liu, J.; Tong, T. Estimating the sample mean and standard deviation from the sample size, median, range and/or interquartile range. BMC Med. Res. Methodol. 2014, 14, 135. [CrossRef] [PubMed]

54. Ferreira, J.M.; Pires, I.M.; Marques, G.; García, N.M.; Zdravevski, E.; Lameski, P.; Flórez-Revuelta, F.; Spinsante, S.; $\mathrm{Xu}, \mathrm{L}$. Activities of Daily Living and Environment Recognition Using Mobile Devices: A Comparative Study. Electronics 2020, 9, 180. [CrossRef]

55. $\mathrm{Ng}, \mathrm{A} . \mathrm{Y}$. Feature Selection, L1 vs. L2 Regularization, and Rotational Invariance. In Proceedings of the Twenty-First International Conference on Machine Learning_ICML '04, Banff, AL, Canada, 4-8 July 2004; ACM Press: Banff, AL, Canada, 2004; p. 78.

56. Zdravevski, E.; Lameski, P.; Mingov, R.; Kulakov, A.; Gjorgjevikj, D. Robust Histogram-Based Feature Engineering of Time Series Data. In Proceedings of the 2015 Federated Conference on Computer Science and Information Systems (FedCSIS), Lodz, Poland, 13-16 September 2015; pp. 381-388.

57. Zdravevski, E.; Lameski, P.; Kulakov, A. Automatic feature engineering for prediction of dangerous seismic activities in coal mines. In Proceedings of the 2016 Federated Conference on Computer Science and Information Systems (FedCSIS), Gdansk, Poland, 11-14 September 2016; pp. 245-248.

58. Pires, I.M.; Santos, R.; Pombo, N.; Garcia, N.M.; Flórez-Revuelta, F.; Spinsante, S.; Goleva, R.; Zdravevski, E. Recognition of activities of daily living based on environmental analyses using audio fingerprinting techniques: A systematic review. Sensors 2018, 18, 160. [CrossRef] [PubMed]

Publisher's Note: MDPI stays neutral with regard to jurisdictional claims in published maps and institutional affiliations.

(C) 2020 by the authors. Licensee MDPI, Basel, Switzerland. This article is an open access article distributed under the terms and conditions of the Creative Commons Attribution (CC BY) license (http://creativecommons.org/licenses/by/4.0/). 